\title{
Antimicrobial Resistance of Microorganisms Causing Pneumonia in Patients of a Public Hospital in Brazilian Pre-Amazon Region
}

\author{
Raíssa G. Assunção ${ }^{1}$, Wellison A. Pereira ${ }^{1}$, Francisco J. R. Nogueira ${ }^{1}$, Itaynara L. Dutra ${ }^{2}$, Tânia M. G. Novais ${ }^{1}$ \\ and Afonso G. Abreu ${ }^{1,2}$ \\ 1. Programa de Pós-Graduação, Universidade CEUMA, São Luís 65075-120, Brazil \\ 2. Programa de Pós-Graduação em Ciências da Saúde, Universidade Federal do Maranhão, São Luís 65080-805, Brazil
}

\begin{abstract}
Nosocomial infections have been common in health services in Brazil. Among them, pneumonia is very highlighted due to the comorbidity that usually affects intensive treatment unit (ITU) patients, and due to the need for endotracheal intubation. The aim of this study was to determine the resistance profile of bacteria isolated from ITU patients with pneumonia in a public hospital in São Luis, an important city in the Brazilian pre-Amazon region. Bacteria strains were obtained from tracheal aspiration and collected for diagnosis as well for phenotypic characterization. Standards culture media, such as blood and MacConkey agar, were used to isolate clinical strains. Automatized Vitek 2 method was used to identify strains and disc diffusion method (Kirby-Bauer) was performed to determine the antimicrobial susceptibility profile. During study, eighty-eight patients were diagnosed with pneumonia, caused by more than 20 different bacteria. Among them, P. aeruginosa (28\%), Acinetobacter baumannii (20\%), Coagulase-negative Staphylococci (19\%), Staphylococcus aureus $(8 \%)$ and Klebsiella pneumoniae (7\%) were the most prevalent microorganisms. Regarding to the resistance, antimicrobial susceptibility test showed that majority of Gram-negative bacteria presented resistance to ampicillin. In Gram-positive bacteria there was a high resistance to ampicillin, penicillin, ciprofloxacin, clindamycin and erythromycin. This study showed a high prevalence of bacteria resistance in ITU patients in a public hospital. Although P. aeruginosa was the most frequent pathogen, it was possible to find other pathogens that were not recurrent, such as Enterobacter spp., Proteus mirabilis and Providencia stuartii. Regarding antimicrobials, the results showed a high resistance to several antimicrobials.
\end{abstract}

Key words: Pneumonia, infection, bacteria resistance.

\section{Introduction}

Respiratory diseases are important worldwide causes of illness. According to the World Health Organization (WHO), 14\% of all deaths in the world have this origin. In 2012, World Health Statistics listed the main potential causes of death in the world, highlighting lower respiratory tract infections, such as pneumonia [1].

Pneumonia is an acute or chronic inflammatory process of the lung parenchyma produced primarily by microorganisms, especially bacteria. After the infection of the pulmonary parenchyma, the

Corresponding author: Afonso G. Abreu, MSc and PhD in Microbiology, research fields: microbiology and immunology. bronchioles and alveoli are filled with inflammatory exudate, hampering hematosis and leading to the classic respiratory insufficiency $[2,3]$.

In general, the most isolated microorganisms from patients with pneumonia are: Staphylococcus aureus, Pseudomonas aeruginosa, Acinetobacter baumannii and Klebsiella pneumoniae [4, 5]. Among the factors that lead to infection, hospitalization for long periods, chronic disease, immunodeficiency, contact with contaminated hospital materials and exposure to antibiotics for a long period are the most common [6]. The risk group is formed by children, elderly (aged 65 and older and hospitalized), pregnant women, immunocompromised, health professionals and patients hospitalized in an Intensive Treatment Unit 
(ITU) [7].

Even with advances in diagnosis and treatment, about 450 million people are still affected every year in the world. In Latin America, up to 1.5 million children under the age of five are infected, leading to the death of approximately 4 million annually [8-10]. Brazil is one of the countries with the highest number of cases [8]. According to the Brazilian information system of the Unified Health System (SUS/DATASUS), between 1996 and 2012, pneumonia was responsible for 37\% of deaths related to the respiratory system [11].

Intravenous endotracheal intubation and mechanical ventilation (MV) have revolutionized the treatment of respiratory failure, however, they can generate negative effects due to the reduction of local defenses against the presence of the endotracheal tube [12, 13]. A survey showed that the incidence of the disease in patients taking MV is up to 21 times higher than in other patients, and that about $20 \%$ of those who use the technique for more than 48 hours will develop the infection [14].

According to the guidelines of the American Thoracic Society, Hospital acquired pneumonia (HAP) and ventilator-associated pneumonia (VAP) remain important causes of morbidity and mortality despite advances in antimicrobial therapy, better supportive care modalities, and the use of a wide-range of preventive measures [15]. HAP is diagnosed after hospitalization and it is estimated that up to $1.1 \%$ of all inpatients develop it, in addition has a low incidence and mortality of up to $50 \%$ [16].

VAP, which develops after 48-72 h of endotracheal intubation, is considered the second most common nosocomial infection and the main cause of death in critically ill patients due to the invasive nature of mechanical ventilation procedures $[17,18]$. It differs from HAP due to its development occurring 48 hours after orotracheal intubation [19,20]. The diagnosis of pneumonia is made through clinical, laboratory and imaging tests, such as radiology. Radiography reveals the presence of persistent or recent progressive infiltrates, pulmonary cavitation or consolidation. The laboratory and clinical exams reveal aspects such as: leukopenia or leukocytosis, fever, purulent secretion with its change or gradual increase [21]. In addition, the microbial identification tests improve the diagnosis and generate relevant information about the resistance profile [21, 22].

The emergence of the theme led the World Health Organization (WHO) to recognize Antimicrobial Resistance as a serious problem faced by global public health [23]. Therefore, control of antimicrobial use during treatment is essential to prevent outbreaks. The aim of this study was to evaluate the antimicrobial resistance profile of microorganisms from patients with pneumonia in a public hospital in Brazil.

\section{Material and Methods}

\subsection{Bacterial Strains}

Samples of tracheal secretion were obtained for convenience from patients attended at a public health hospital with diagnosis of pneumonia, this study is a retrospective analysis which was conducted with authorization of the Laboratory of Microbiology. The results were obtained from a data bank, as well as approved by the Research Ethics Committee, in a period of four months, in São Luis, Brazil. After collection, conventional culture media were used for sowing of samples, such as blood agar, MacConkey agar and Thioglycolate broth. All isolates were identified using conventional techniques [24] and the Vitek 2 system, an automated assay (BioMérieux®, Marcy l'Etoile, France).

\subsection{Antimicrobial Susceptibility Tests}

Susceptibility testing was performed and interpreted via Kirby-Bauer method, as recommended by the Clinical and Laboratory Standards Institute (CLSI), and the Vitek 2 system.

The following antimicrobials were used for the test: amikacin, amoxicillin/clavulanate, ampicillin, ampicillin/sulbactam, amphotericin, aztreonam, 
cefazolin, cefepime, cefotaxime, cefoxitin, ceftazidime, ceftriaxone, cefuroxime, ciprofloxacin, clindamycin, chloramphenicol, cotrimoxazole, erythromycin, ertapenem, fluconazole, gentamycin, imipenem, levofloxacin, linezolid, meropenem, nitrofurantoin, norfloxacin, oxacillin, penicillin, polymyxin $\mathrm{B}$, teicoplanin, tetracycline, ticarcillin/clavulanate, tobramycin, vancomycin, minocycline, gentamicin, streptomycin, rifampicin, tigecycline, colistin, cefuroxime, ofloxacin.

\section{Results}

In this study, a total of eighty-eight patients were diagnosed with pneumonia, of which 51 were male $(57.95 \%)$ and $37(42.04 \%)$ were female. The majority of patients were elderly (aged 60 or over) $(n=47)$, followed by adult $(n=41)$. After the microbiological tests, it was possible to identify twenty different species of bacteria involved in cases of pneumonia (Table 1). The most frequent were $P$. aeruginosa $(n=$ 29), A. baumannii $(n=21), S$. aureus $(n=8)$ and $K$. pneumoniae $(n=7)$, as shown in Table 1 . The antimicrobial susceptibility test showed that the most of the Gram-negative bacteria presented resistance to ampicillin, ampicillin/sulbactam. Some isolates showed resistance to cephalosporins, gentamicin, levofloxacin, imipenem and meropenem (Table 2). Gram-positive bacteria presented a high resistance to ampicillin, ciprofloxacin, clindamycin, erythromycin and oxacillin (Table 3).

\section{Discussion}

Bacterial resistance to antimicrobials has been widely discussed as an important problem in ITU worldwide. The main factor that leads to colonization and/or infection caused by resistant microorganisms is the inadequate use of antimicrobials.

All antibiotics in some way promote selection and resistance, not only by molecular mechanisms, but mainly by elimination of the sensitive microbiota and its substitution by resistant microorganisms [25]. The cause of emergence and spread of multidrug-resistant bacteria in hospitals is multifactorial and includes mainly the abuse of antimicrobials. Infections associated with virulent pathogens have become increasingly difficult to administer and often require higher doses, favoring increased resistance [26].

The results found in this study showed a high microbial resistance to the most antibiotics tested, and presented quite similarity when compared to the data reported by Ju et al. [27], Varon-Vega et al. [28] and Seibert [29], since the most prevalent gender was the

Table 1 Microorganisms isolated from patients diagnosed with pneumonia in a public hospital in São Luís, Brazil.

\begin{tabular}{lll}
\hline Microorganism & No. & $\%$ \\
\hline Pseudomonas aeruginosa & 29 & 28.1 \\
Acinetobacter baumannii & 21 & 20.4 \\
Coagulase-negativa Staphylococcus & 20 & 19.4 \\
Staphylococcus aureus & 8 & 7.8 \\
Klebsiella pneumoniae & 7 & 6.8 \\
Enterobacter spp. & 4 & 3.8 \\
Enterococcus spp. & 4 & 3.9 \\
Serratia marcescens & 2 & 1.9 \\
Stenotrophomonas maltophilia & 2 & 1.9 \\
Achromobacter xylosoxidans & 1 & 1.0 \\
Elizabethkingia meningoseptica & 1 & 1.0 \\
Morganella morganii & 1 & 1.0 \\
Proteus mirabilis & 1 & 1.0 \\
Providencia stuartii & 1 & 1.0 \\
Streptococcus pneumoniae & 1 & 1.0 \\
\hline Total & 103 & 100 \\
\hline
\end{tabular}


Table 2 Resistance profile of the main Gram-negative bacteria isolated from patients diagnosed with pneumonia in a public hospital in São Luís, Brazil.

\begin{tabular}{|c|c|}
\hline Gram-negative bacteria /Antimicrobial & Resistance $(\%)$ \\
\hline \multicolumn{2}{|l|}{ Serratia marcescens } \\
\hline Ampicillin, ampicillin /sulbactam & $100 \%$ \\
\hline Cefoxitin & $50 \%$ \\
\hline \multicolumn{2}{|l|}{ Pseudomonas aerugiosa } \\
\hline Imipenem & $48.20 \%$ \\
\hline Meropenem & $27.50 \%$ \\
\hline Ciprofloxacin & $17.20 \%$ \\
\hline Cefepime, levofloxacin & $10.30 \%$ \\
\hline Ceftazidime & $6.90 \%$ \\
\hline Gentamicin & $3.40 \%$ \\
\hline \multicolumn{2}{|l|}{ Proteus mirabilis } \\
\hline Ampicillin, ampicillin/sulbactam, cefotaxime, cefoxitin, ceftazidime, ceftriaxone & $100 \%$ \\
\hline \multicolumn{2}{|l|}{ Acinetobacter baumanii } \\
\hline Cefepime & $81 \%$ \\
\hline Meropenem & $76.19 \%$ \\
\hline Ciprofloxacin, imipenem & $76.20 \%$ \\
\hline Cefriaxone & $61.90 \%$ \\
\hline Cefotaxime & $57.10 \%$ \\
\hline Ampicillin/sulbactam, ceftazidime & $47.60 \%$ \\
\hline Levofloxacin & $42.90 \%$ \\
\hline Gentamicin & $33.30 \%$ \\
\hline Ampicillin & $28.60 \%$ \\
\hline Cefoxitin & $14.30 \%$ \\
\hline \multicolumn{2}{|l|}{ Klebsiella pneumonia } \\
\hline Ampicillin & $100 \%$ \\
\hline Ampicillin/sulbactam, cefepime, cefotaxime, ciprofloxacin, imipenem & $14.30 \%$ \\
\hline
\end{tabular}

masculine. On the other hand, in a study conducted by Bezerra et al. [14] that followed 74 patients, the majority of them was women.

The main microorganisms found here were $P$. aeruginosa, corroborating with the findings of Pires et al. [30], that conducted a retrospective study based on microbiological data obtained from the record book at Laboratory of Bacteriology, Hospital das Clínicas, Pernambuco, Brazil. Regarding the use of antimicrobials, there was a divergence, since here ciprofloxacin and imipenem were more effective in relation to $P$. aeruginosa. On the other hand, in a retrospective study conducted at Zhongshan Hospital of Fudan University in Shanghai, HAP was caused mainly by $A$. baumannii [27]. Among the antimicrobials tested, cefotaxime and meropenem presented similar results with our study, since $A$. baumannii was also resistant to these drugs. Among the Enterobacteriaceae family, $K$. pneumoniae was the most frequent, presenting a total resistance against ampicillin. This same pathogen was the most prevalent among the bacteria in a descriptive study carried out by Seibert [29]. Similar to this study carried out in São Luís, Barnes et al. [31] analyzed 226 patients with nosocomial pneumonia and numerous types of bacteria were found, among them: A. baumannii, $K$. pneumoniae, S. marcescens, $P$. mirabilis, among others.

The bacterial species reported in our study are recurrent in several studies, however, in the study by Patil et al. [32], K. pneumoniae was more prevalent. In addition, with regard to its antimicrobials, meropenem was successfully used in the treatment Acinetobacter spp. and Pseudomonas spp. This shows that knowledge 
Table 3 Resistance profile of the main Gram-positive bacteria isolated from patients diagnosed with pneumonia in a public hospital in São Luís, Brazil.

\begin{tabular}{|c|c|}
\hline Gram-positive bacteria/antimicrobial & Resistance $(\%)$ \\
\hline \multicolumn{2}{|l|}{ Staphylococcus aureus } \\
\hline Ampicillin, erythromycin, penicillin & $75 \%$ \\
\hline Ciprofloxacin, clindamycin, oxacillin & $50 \%$ \\
\hline Levofloxacin & $37.50 \%$ \\
\hline Cephalothin & $25 \%$ \\
\hline Gentamicin & $12 \%$ \\
\hline \multicolumn{2}{|l|}{ Staphylococcus epidermidis } \\
\hline Ampicillin, ciprofloxacin, clindamycin, erythromycin, oxacillin, penicillin & $100 \%$ \\
\hline Cephalothin, levofloxacin & $50 \%$ \\
\hline \multicolumn{2}{|l|}{ Staphylococcus haemolyticus } \\
\hline Ampicillin, ciprofloxacin, clindamycin, erythromycin, oxacillin, penicillin & $100 \%$ \\
\hline \multicolumn{2}{|l|}{ Streptococcus pneumoniae } \\
\hline Ciprofloxacin, tetracycline & $100 \%$ \\
\hline Ampicillin, erythromycin & $50 \%$ \\
\hline \multicolumn{2}{|l|}{ Staphylococcus coagulase negativa } \\
\hline Ampicillin, penicillin & $100 \%$ \\
\hline Erythromycin & $88.20 \%$ \\
\hline Ciprofloxacin, oxacillin & $70.50 \%$ \\
\hline Clindamycin, levofloxacin & $64.70 \%$ \\
\hline Cephalothin & $58.80 \%$ \\
\hline Tetracycline & $29.40 \%$ \\
\hline Rifampicin & $23.60 \%$ \\
\hline Gentamicin & $17.60 \%$ \\
\hline \multicolumn{2}{|l|}{ Enterococcus spp. } \\
\hline Ciprofloxacin, oxacillin, rifampicin, tetracycline & $25 \%$ \\
\hline
\end{tabular}

of the susceptibility pattern of local pathogens will define the strategy in the use of antimicrobials. In the present study, it was also possible to observe that the most frequent Gram-positive bacteria was Staphylococcus spp., mainly $S$. aureus. These data corroborated with the study by Walter et al. [4]. Besides that, this microorganism presented a high resistance to erythromycin followed by oxacillin, corroborating a study reported in Korea by Lee and colleagues [33] with patients who had pneumonia.

Among the Enterobacteriaceae family, $K$. pneumoniae was the main isolated. Its resistance is associated with the production of $\beta$-lactamases, among other mechanisms. Here it was possible to observe a high resistance to beta-lactams, followed by carbapenems, as well as a study conducted by Montufar-Andrade et al. [34]. P. aeruginosa and $A$. baumannii were the main microorganisms found in this study. Regarding the use of antimicrobials, carbapenems had a high rate of resistance in these bacteria, therefore a high consumption of imipenem/meropenem may play an important role in the selective pressure exerted by antibiotics in these strains. Moreira et al. [35] also observed high rates of resistance to carbapenems.

In this way, to avoid the respiratory infection, Almeida et al. [36] after conducting a study at university hospital in the city of Recife, Brazil, listed some preventive measures to prevention. These include: $30-45^{\circ}$ head elevation, discontinuation of sedation, oral hygiene of the patient and cuff pressure. Together, these actions can culminate in improving the patient's health status. It is important to note that most of the reviews and studies presented as the most recurrent microorganisms the same ones that were identified in this research, as well as the resistance profile despite 
small variations. These studies have in common the high rates of bacterial resistance.

\section{Conclusions}

Pneumonia is one of the infectious diseases that kill the most in the world and Brazil is among the countries with the highest incidence of this disease, which is even more serious among patients who use mechanical ventilation. Although $P$. aeruginosa was the most isolated pathogen in this work, the presence of other pathogens, such as A. baumannii and Staphylococcus spp. were frequent. Regarding antimicrobials, the results showed that microorganisms presented a high resistance, a matter of concern, mainly due to the high morbimortality associated with these phenomena.

\section{Acknowledgements}

We would like to thank the Foundation for Research Support and Scientific and Technological Development of Maranhão (FAPEMA) and CEUMA University.

\section{References}

[1] Ferraz, R. O., Oliveira-friestino, J. K., and Francisco, P. M. S. B. 2017. "Tendência de mortalidade por pneumonia nas regiões brasileiras no período entre 1996 e 2012.” J. Bras. Pneumol. 43 (4): 274-9.

[2] Matoso, L. M. L., and de Castro, C. H. A. 2013. "Indissociabilidade clínica e epidemiológica da pneumonia." CATUSSABA 2 (2): 11-24.

[3] Machado, D., de Moraes, L. T. A. R., de Oliveira, M. E., and Vianna, I. A. 2017. "Pneumonia: Tratamento e Evolução." Cad. UniFOA 5 (14): 65-9.

[4] Walter, J., Haller, S., Quinten, C., Kärki, T., Zacher, B., and Eckmanns, T. 2018. "Healthcare-Associated Pneumonia in Acute Care Hospitals in European Union/European Economic Area Countries: An Analysis of Data from a Point Prevalence Survey, 2011 to 2012.” Euro Surveill. 23 (32): 1700843.

[5] Medell, M., et al. 2012. "Clinical and Microbiological Characterization of Pneumonia in Mechanically Ventilated Patients." Braz. J. Infect. Dis. 16 (5): 442-7.

[6] Perez, M. G., et al. 2017. "Factores de riesgo de focos secundarios de infección en ninos con bacteriemia por Staphylococcus aureus adquirida en la comunidad, estudio de cohorte 2010-2016." Enferm. Infecc. Microbiol. Clin.
[7] Brazil. Ministry of Health. Secretariat of Health Surveillance. 2010. "Department of Epidemiological Surveillance. Infectious and Parasitic Diseases: Pocket Guide." Ministry of Health. Secretariat of Health Surveillance, Department of Epidemiological Surveillance. 8: 444.

[8] Nunes, S. E. A., Minamisava, R., Vieira, M. A., Itria, A., Pessoa, V. P., de Andrade, A. L. S. S., and Toscano, C. M. 2017. "Hospitalization Costs of Severe Bacterial Pneumonia in Children: Comparative Analysis Considering Different Costing Methods.” Einstein 15 (2): 212-9.

[9] Rocha Neto, O. G., Leite, R. F., and Baldi, B. G. 2013. “Atualização em pneumonia comunitária viral." Rev. Assoc. Med. Bras. 59 (1): 78-84.

[10] Ruuskanen, O., Lahti, E., Jennings, L. C., and Murdoch, D R. 2011. "Viral Pneumonia." The Lancet 377 (9773): 1264-75.

[11] Sociedade de Pediatria do Rio Grande do Sul. 2014. "Porto Alegre: a Sociedade. Informe Técnico." Campanha Nacional de Vacinação Contra a Influenza.

[12] Albernaz, M. P., Gonçalves, E. O., Lima, M. S., Lima Melo, J., Pontes, M. S. R., and Sousa, A. O. B. 2015. "Nursing Care Practices and Prevention of Pneumonia Associated with Mechanical Ventilation in ICU." Revista de enfermagem UFPE 9 (12): 1069-77.

[13] Silva, R. M. 2011. "Pneumonia Associated with Mechanical Ventilation: Risk Factors." Rev. Bras. Clin. Med. 9 (1): 5-10.

[14] Bezerra, E. L., Lima, A. I. F., Nbrebre, A. R. R., et.al. 2012. "Prevalence of Pneumonia in Patients of an Intensive Care Unit of a School Hospital in Fortaleza, CE". Rev. Bras. Promoç. Saúde 25 (2): 20-4.

[15] American Thoracic Society, Infectious Diseases Society of America. 2005. "Guidelines for the Management of Adults with Hospital-Acquired, Ventilator-Associated, and Healthcare-Associated Pneumonia." Amer. Jour. Respir. Cri. Medic. 171 (4): 388-416.

[16] Rufino, R., Pugliese, J. G., Kirk, K., Bartholo, T. G. P., and Mafort, T. T. 2010. "Pneumonia Acquired at the Hospital-Critical View." Rev. Hosp. Univ. Pedro Ernesto 9 (2): 30-40.

[17] Timsit, J. F., Esaied, W., Neuville, M., Bouadma, L., and Mourvllier, B. 2017. "Update on Ventilator-Associated Pneumonia.” Research 6: 2061

[18] Rodrigues, P. M. A., et al. 2009. "Pneumonia Associated with Mechanical Ventilation: Epidemiology and Impact on the Clinical Evolution of Patients in an Intensive Care Unit.” J. Bras. Pneumol. 35 (11): 1084-91.

[19] Ibn Saied, W., et al. 2017. "Impact Regarding the Implementation of Strategies for Prevention, Colonization with Multi-resistant Bacteria and Use of Antimicrobials on the Risk of VAP Start and Afternoon: An Analysis of the 
a Public Hospital in Brazilian Pre-Amazon Region

OUTCOMEREA Network." PLoS ONE 12 (11): e0187791.

[20] Amaral, S. M., Cortes, A. Q., and Pires, F. R. 2009. "Nosocomial Pneumonia: Importance of the Oral Microenvironment.” J. Bras. Pneumol. 35 (11): 1116-24.

[21] Dalmora, CH. et al. 2013. "Defining Pneumonia Associated with Mechanical Ventilation: Concept in (de) Construction". Brazilian Journal of Intensive Care Therapy 25 (2): 81-6.

[22] Bonten, M. J. M. 2011. "Ventilator Associated Pneumonia: Preventing the Inevitable." Clinical Infections Diseases 52 (1): $115-21$

[23] Soldatelli, R. P. P., and Lorenzini, E. 2014. "Strategies for Prevention of Bacterial Resistance: Contributions to Patient Safety." Revista Cuidarte 5 (2): 757-64.

[24] Clinical Laboratories Standards Institute. 2015. Performance Standards for Antimicrobial Susceptibility Testing. CLSI. Wayne. PA.

[25] Langdon, A., Nathan C., and Gautam, D. 2018. "The Effects of Antibiotics in the Microbiome during Development and Alternative Approaches to Modulation Therapy." Genome Medicine 8: 39.

[26] Pietropaolo, A., Jones, P., Moors, M., Birch, B., and Somani, B. K. 2018. "Use and Effectiveness of Antimicrobial Intravesical Treatment for Prophylaxis and Treatment of Recurrent Urinary Tract Infections (UTIs): A Systematic Review." Curr Urol Rep. 19 (10): 78.

[27] Ju, M., et al. 2018. "Fatores de risco para mortalidade em pacientes internadosem UTI com Pneumonia Associada à Ventilação Mecânica de Acinetobacter Baumannii: Impacto da Citotoxicidade Bacteriana." Journal of Thoracic Disease 10 (5): 2608-17.

[28] Varon-vega, F. A., et al. 2017. "Traqueobronquitis y neumonía asociadas a ventilación mecánica en unidades de cuidado intensivo de Latinoamérica: epidemiología, curso clínico y desenlaces.” Infect. 21 (2): 74-80.
[29] Seibert, G., et al. 2014. "Hospital Infections Caused by Enterobacteriaceae Producing Klebsiella Pneumoniae Carbapenemase in a Hospital School.” Einstein 12 (3): 282-6.

[30] Pires, E. J. V. C., et al. 2009. “Análise epidemiológica de isolados clínicos de Pseudomonas aeruginosa provenientes de hospital universitário." Rev. Bras. Ter. Intensiva 21: 384-90.

[31] Barnes, A., Bergan, E. H., Tura, B. R., and Lamas, C. C. 2014. "Impact of Improvement in Preoperative Oral Health on Nosocomial Pneumonia in a Group of Cardiac Surgery Patients: A Single Arm Prospective Intervention Study." Intensive Care Med. 40 (4): 23-31.

[32] Patil, H. V., and Patil, V. C. 2017. "Incidence, Bacteriology, and Clinical Outcome of Ventilator-Associated Pneumonia at Tertiary Care Hospital." Journal of Natural Science, Biology, and Medicine 8 (1): 46-55.

[33] Lee, Y., Hong, S. K., Choi, S., Im, W., Yong, D., and Lee, K. 2015. "In Vitro Activity of Tedizolid against Gram-Positive Bacteria in Patients With Skin and Skin Structure Infections and Hospital-Acquired Pneumonia: A Korean Multicenter Study." Annals of Laboratory Medicine 35 (5): 523-30.

[34] Montufar-andrade, F. E. 2016. "Experiências clínicas com infecções causadas por Klebsiella pneumoniae productora de carbapenemasa, em uma instituição de saúde universitária em Medellín, Colômbia.” Infect. 20 (1): 17-24.

[35] Moreira, M. R., et al. 2013. "Antimicrobial Use, Incidence, Etiology and Resistance Patterns in Bacteria Causing Ventilator-Associated Pneumonia in a Clinical-Surgical Intensive Care Unit." Rev. Soc. Bras. Med. Trop., Uberaba 46 (1): 39-44.

[36] Almeida, K. M. V., et al. 2015. "Sobre a Pneumonia." Revista de Enfermagem da UFSM 5 (2): 247-56. 